\title{
Archives Of Breast

\section{The Importance of Metastatic Papillary Serous Carcinoma in Cases of High-grade Encapsulated Papillary Carcinoma of the Breast: An Extremely Rare Case and a Possible Pitfall}

Helen J Trihia*a, Gabriela Stanc ${ }^{a}$, Nikolaos Charalampakis ${ }^{b}$, Argyri Kayiac ${ }^{c}$, Dimitrios Kouzos ${ }^{a}$, Eleftheria Ignatiadou ${ }^{\mathrm{d}}$, Kassiani Manoloudaki ${ }^{\mathrm{e}}$, Ioannis Provatas ${ }^{\dagger}$

aDepartments of Pathology, Metaxas Memorial Cancer Hospital, Piraeus, Greece

${ }^{b} M e d i c a l$ Oncology, 'Metaxas' Memorial Cancer Hospital, Piraeus, Greece

'Nuclear Medicine, 'Metaxas' Memorial Cancer Hospital, Piraeus, Greece

${ }^{d}$ Breast Unit, 'Metaxas' Memorial Cancer Hospital, Piraeus, Greece

ePathology, Tzanion Hospital, Piraeus, Greece

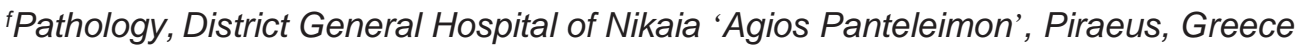

\section{ARTICLE INFO}

Received:

22 September 2021

Revised:

01 January 2022

Accepted:

03 January 2022

Keywords:

encapsulated papillary

carcinoma,

metastasis,

breast cancer,

peritoneal serous

carcinoma

Copyright $\odot$ 2022. This is an open-access article distributed under the terms of the Creative Commons Attribution-Non-Commercial 4.0 International License, which permits copy and redistribution of the material in any medium or format or adapt, remix, transform, and build upon the material for any purpose, except for commercial purposes.

\section{ABSTRACT}

Background: Encapsulated papillary carcinoma (EPC) of the breast has a favorable prognosis. High-grade EPCs, triple-negative or HER-2-positive, are dealt with as invasive carcinomas. Breast metastasis associated with serous carcinoma is a late-stage event. The discrimination between the two diagnoses can be very challenging.

Case Presentation: A 79-year-old woman with a history of well controlled high-grade serous papillary carcinoma of the peritoneum went through a total left mastectomy and sentinel lymph node biopsy (SLNB) because of an invasive carcinoma in her left breast. In the lab, a peripheral nodular mass of $4 \mathrm{~cm}$ was found. Microscopically, large intracystic papillary stalks, with high nuclear grade, surrounded by collagenous tissue were identified compatible with invasive encapsulated papillary carcinoma with positive estrogen receptor. A few months later, the patient was diagnosed with a supraclavicular cervical mass, which on fine needle biopsy (FNB) was indicative of metastatic serous papillary carcinoma. Immunohistochemical stains were similar in breast and previously treated peritoneal tumor showed ER+, PAX8+, p53+ (wild type) and high Ki-67 (80\%). WT1 was positive only in peritoneal serous carcinoma. GATA-3 was weakly, scarcely expressed in both specimens. The findings pointed to metastatic serous papillary carcinoma (SPC) in the breast, mimicking primary carcinoma of the EPC type.

Conclusion: Pathology of breast metastases and distinction from primary breast cancers is done by a combination of morphological and IHC features. In our case, the lack of clinical history, the type of surgical approach (mastectomy and SLNB), the solitary lesion, the EPC pattern of growth and the diffuse ER+ staining, were indicative of primary breast lesion. Various morphologic growth patterns of metastatic PSC have been described, among which EPC-like needs to be considered.

\author{
*Address for correspondence: \\ Helen J Trihia, MD \\ Department of Pathology, Metaxas Memorial Cancer \\ Hospital, Piraeus, Greece \\ Tel: +306970918556 \\ Email: eltrix@otenet.gr
}

\section{INTRODUCTION}

Encapsulated papillary carcinoma (EPC) is a rare type of breast carcinoma, showing fine (delicate) fibrovascular stalks covered by neoplastic epithelial cells of low or intermediate nuclear grade, typically present within a cystic space and surrounded by a 
fibrous capsule. There are usually no myoepithelial cells (ME) at the periphery of the lesion. ${ }^{1}$ Less often, it is composed of an aggregate of close nodules. ${ }^{1}$ Lesions presenting with a growth pattern similar to that of EPC but with nuclear pleomorphism and increased mitotic activity, and/or a triple negative or HER2-positive phenotype, should be graded, staged, and managed as invasive breast carcinomas (IBCs). ${ }^{2,3}$ Encapsulated papillary carcinomas (EPC) are found most frequently in elderly women, and are usually asymptomatic, or presented as a palpable subareolar mass and/or nipple discharge. ${ }^{1}$

Metastasis of ovarian or peritoneal serous carcinoma to the breast and/or axillary lymph nodes is rare and is as a late phenomenon associated with the development of disease at multiple sites. ${ }^{4,5,6}$ Secondary breast malignancies are rare representing $<1 \%$ of all breast tumors, but their recognition and distinction from mammary carcinoma are important because the treatment and prognosis differ significantly. When clinical history is not known at the time of diagnosis, both clinical and pathological misdiagnoses are possible, especially when the histological growth pattern can mimic a primary breast carcinoma.

We report a 79-year-old lady, with past history of high-grade serous carcinoma of the peritoneum (HG-
SCP) which presented with high-grade EPC of the breast seven years later.

\section{CASE PRESENTATION}

A 79-year-old woman was referred to our hospital for a lump of her left breast. The radiological appearances were of an ovoid, circumscribed, nodular, lobulated, deeply seated, solid mass, of approximately $4 \mathrm{~cm}$, in the upper outer quadrant of the left breast. There were also intra-mammary lymph nodes in both breasts. Preoperative diagnosis was made based on fine needle aspiration (FNA) findings (malignant cells present, consistent with adenocarcinoma of the breast). After discussion of the diagnosis and according to the patient's desire, total mastectomy and Sentinel Lymph Node Biopsy (SL-NB) were performed based on the results of triple test (clinical, radiological and cytological characteristics). On gross examination, the excised specimen measured $28 \times 16,5 \times 0,5 \mathrm{~cm}$. Cut sectioning showed a peripheral circumscribed tumor of $4 \times 2 \times 2 \mathrm{~cm}, 9 \mathrm{~cm}$ from the nipple, in the upper outer quadrant of the breast. Histopathological examination showed a tumor arranged in papillary stalks within cystic spaces, surrounded by fibrous tissue (Figures 12).

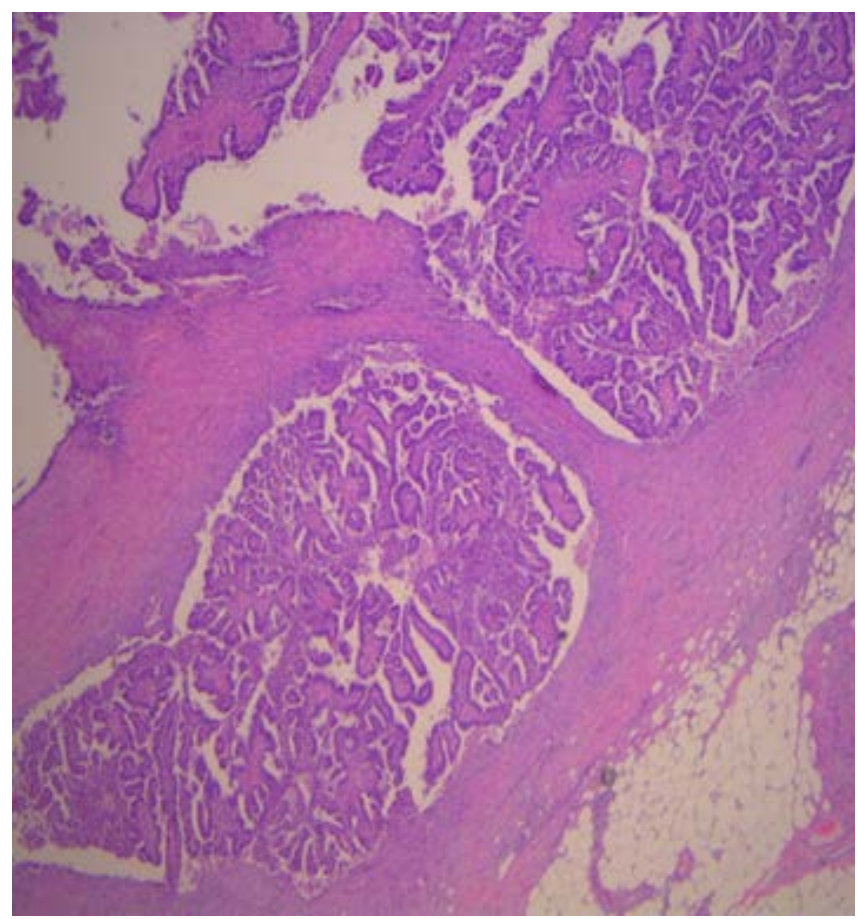

Figure 1. a. X25, b. X100: H\&E (heamatoxylin \& eosin). Tumor architecture. Breast tumor with EPC-like pattern of growth. Tumor arranged in papillary stalks within cystic spaces, surrounded by fibrous tissue. Circumscribed pushing infiltrative pattern. In (a) breast parenchyma on the left of the tumor is also included to denote the location of the tumor in the breast 

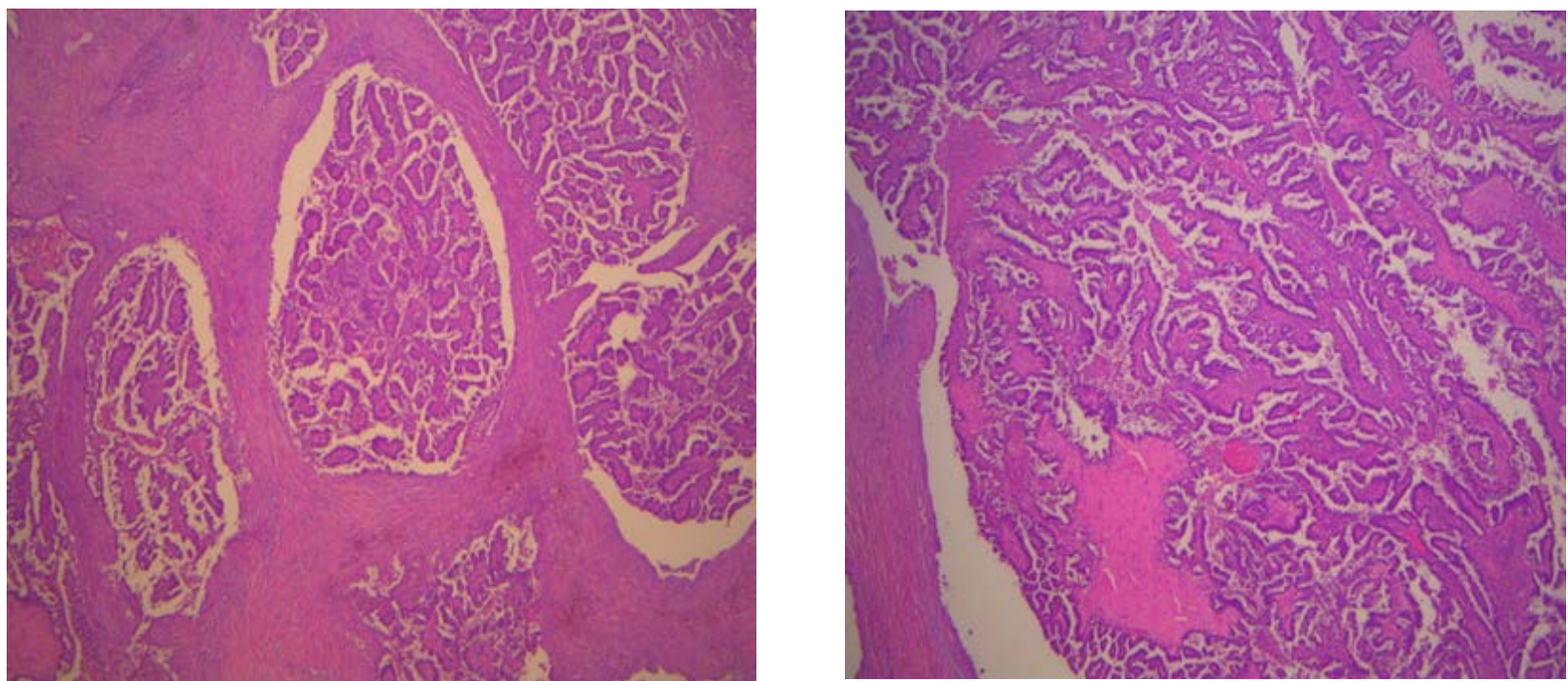

Figure 2. a. X100, b. X200: H\&E. As in 1. Intracystic papillary stalks, within multiple cystic spaces, slender and in areas more thickened, with hyalinized cores

There were minor microscopic necrotic areas and scattered psammoma-like calcifications in the stroma and in the tumor (Figure 3). On close inspection, the cells showed high nuclear grade and a very high mitotic rate (Figure 4).

There were also hemosiderin pigment granules and sparse inflammatory cells, at the periphery of the cystic spaces. Staining for myoepithelial markers (SMA, p63, CK14), demonstrated the absence of myoepithelial cells at the periphery of the lesion. In addition, there was a positive expression for hormone receptors, ER and PR ( $>50 \%)$, lack of ERBB2 (HER2) gene amplification and increased Ki-67

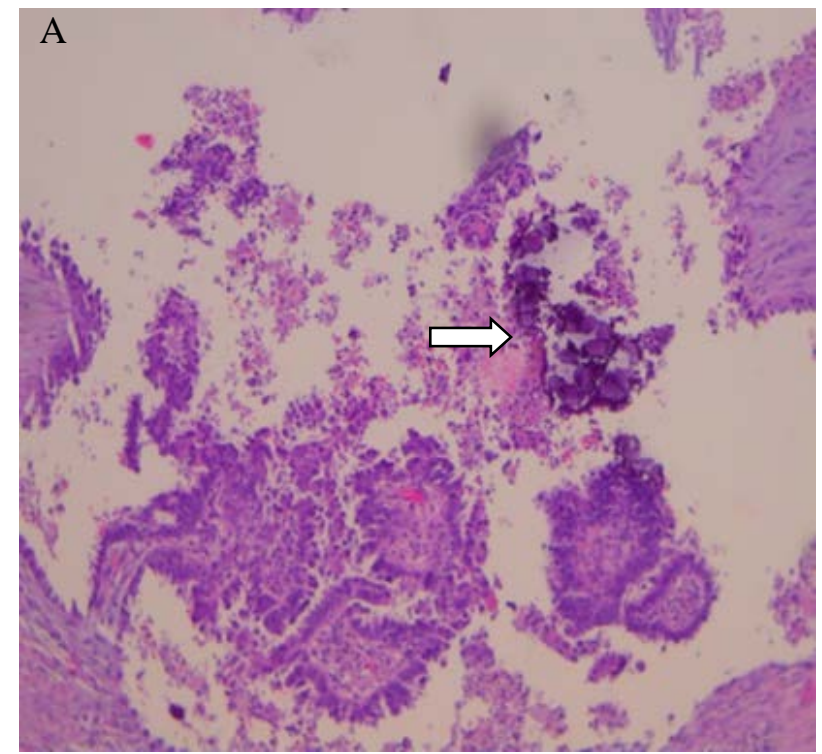

proliferation index (approximately 80\% of the cells). There were no abnormalities in the surrounding breast parenchyma. SLNB was unremarkable. Final histopathological diagnosis was of an invasive encapsulated papillary carcinoma (EPC) of the breast, mainly due to the high cytonuclear features and increased mitotic activity, although no definite evidence of stromal invasion was recognized. The papillary architecture with well-circumscribed pushing margins was indicative of a less infiltrative nature with a subsequently lower metastatic potential. The patient was further referred for hormone treatment (letrozole for 5 years).

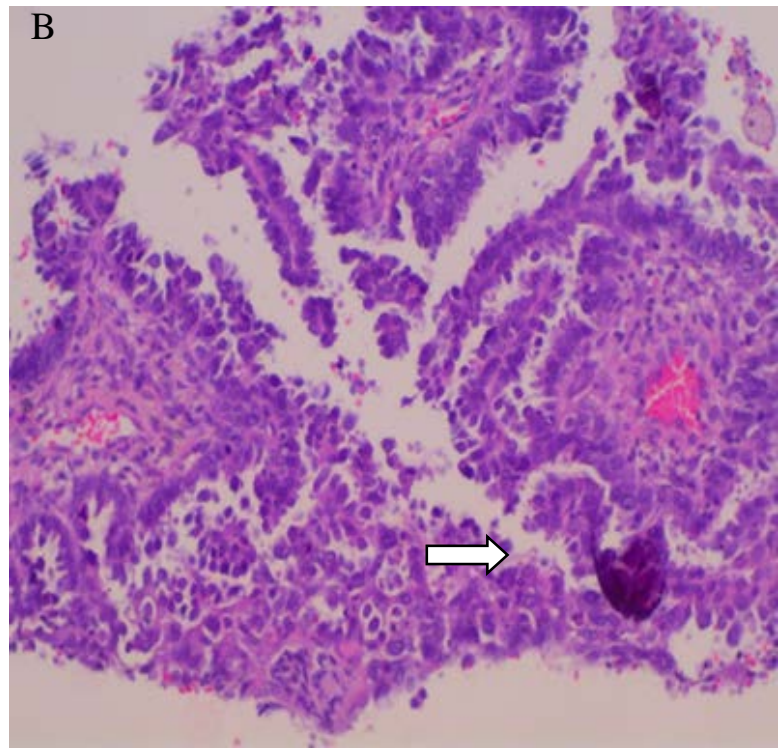

Figure 3. a. X100, b. X200: H\&E. Obvious psammoma-like calcifications in tumor papillae (arrows) 

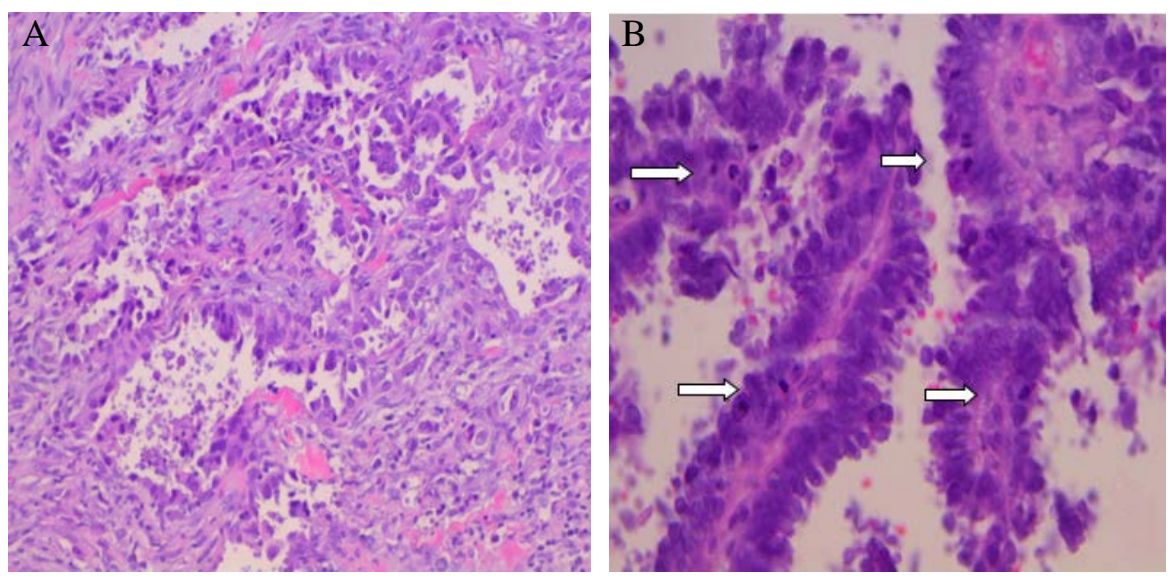

Figure 4. a. X 200, b. X400: H\&E. Micropapillary growth pattern (a) with high nuclear grade and brisk mitotic activity (arrows in $\mathbf{b}$ )
A few months after mastectomy, a 'left supraclavicular, cervical mass-FNB biopsy’ of approximately $5 \mathrm{~cm}$ in diameter (Figure 5) was excised and sent to the lab with the clinical diagnosis of metastatic carcinoma. On retrospective review of the clinical history, the patient had a history of total abdominal hysterectomy with bilateral salpingo-oophorectomy and omentectomy seven years ago with the diagnosis of an extraovarian, primary high grade serous carcinoma of the peritoneum, with a hierarchical papillary, tubular and solid growth pattern (Figure 6) and psammoma bodylike calcifications (Figure 6). Immunohistochemically, the peritoneal tumor showed positive expression for CK7, CA125, CK5/6 (focally), EMA, Tag72 (rare), PLAP, WT1 (Figure 7), PAX8, p53, BerEP4 (mild), ER and PR. The patient had a favorable response to platinum-based chemotherapy and cytoreductive surgery during these 7 years. At the time of diagnosis, there were no radiological findings of local breast or axillary lymph node recurrence.

The review of the breast carcinoma diagnosis was made and IHC studies were undertaken for GATA-3, mammaglobin, GCDFP-15, WT1 and PAX8, which showed negative expression for mammaglobin, GCDFP-15 and WT1, positive expression for p53 and PAX8 (Figure 8) and focal positive expression for
GATA-3 (scarce positive cells). By revision of both specimens of peritoneum and breast, the diagnosis of metastatic papillary serous carcinoma was made and the patient continued the treatment with chemotherapy. She received three cycles of chemotherapy with a combination of carboplatin and paclitaxel, administered every 3 weeks. Unfortunately, after the third course of chemotherapy the patient was deceased because of acute rupture of abdominal aortic aneurysm.

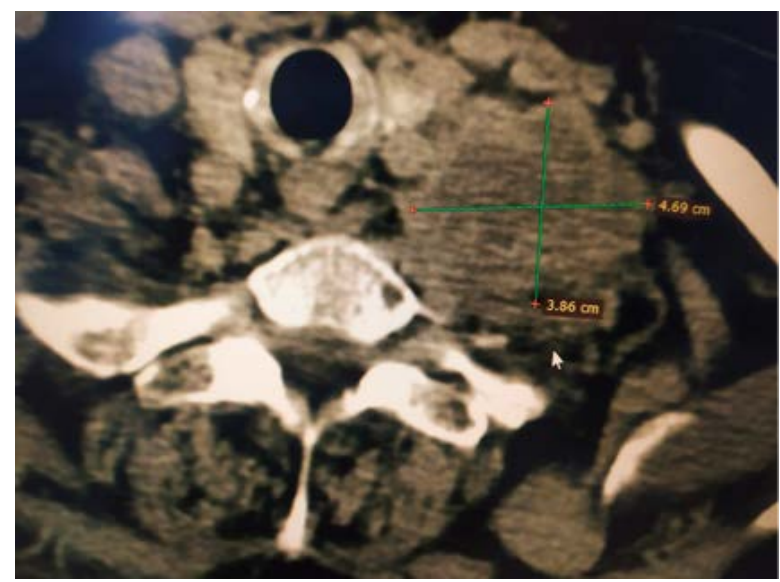

Figure.5. CT imaging of left supraclavicular mass
Figure 6. a. X25, b. X100: H\&E. Peritoneal serous carcinoma with hierarchical (small nested and slit-like) papillary pattern of growth and obvious psammoma body-like calcifications (arrows)
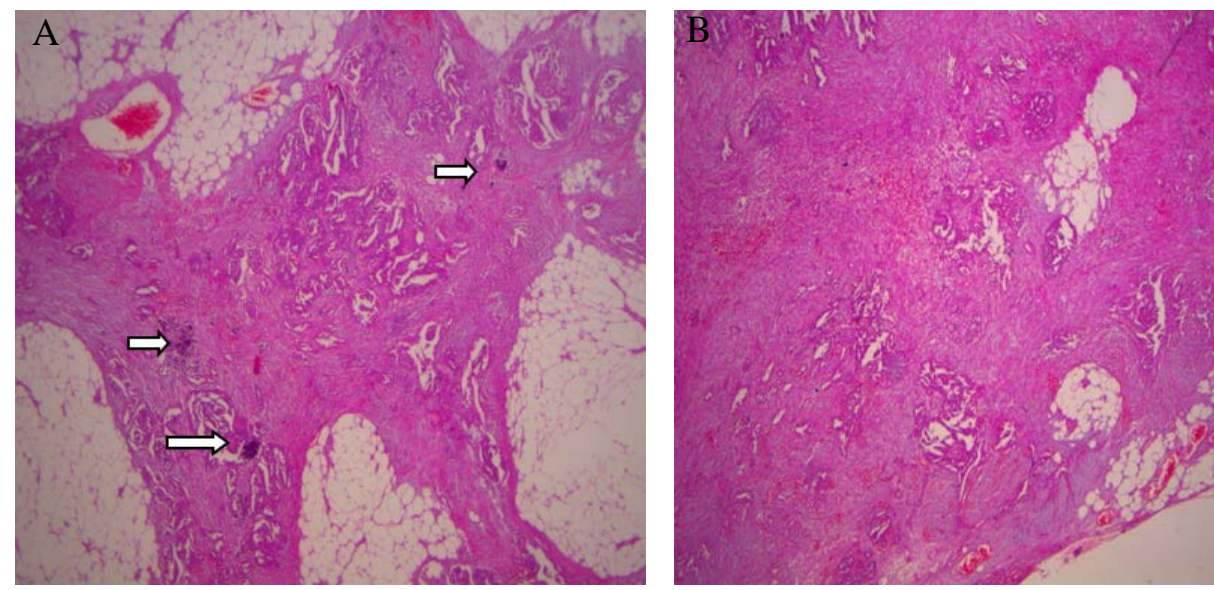

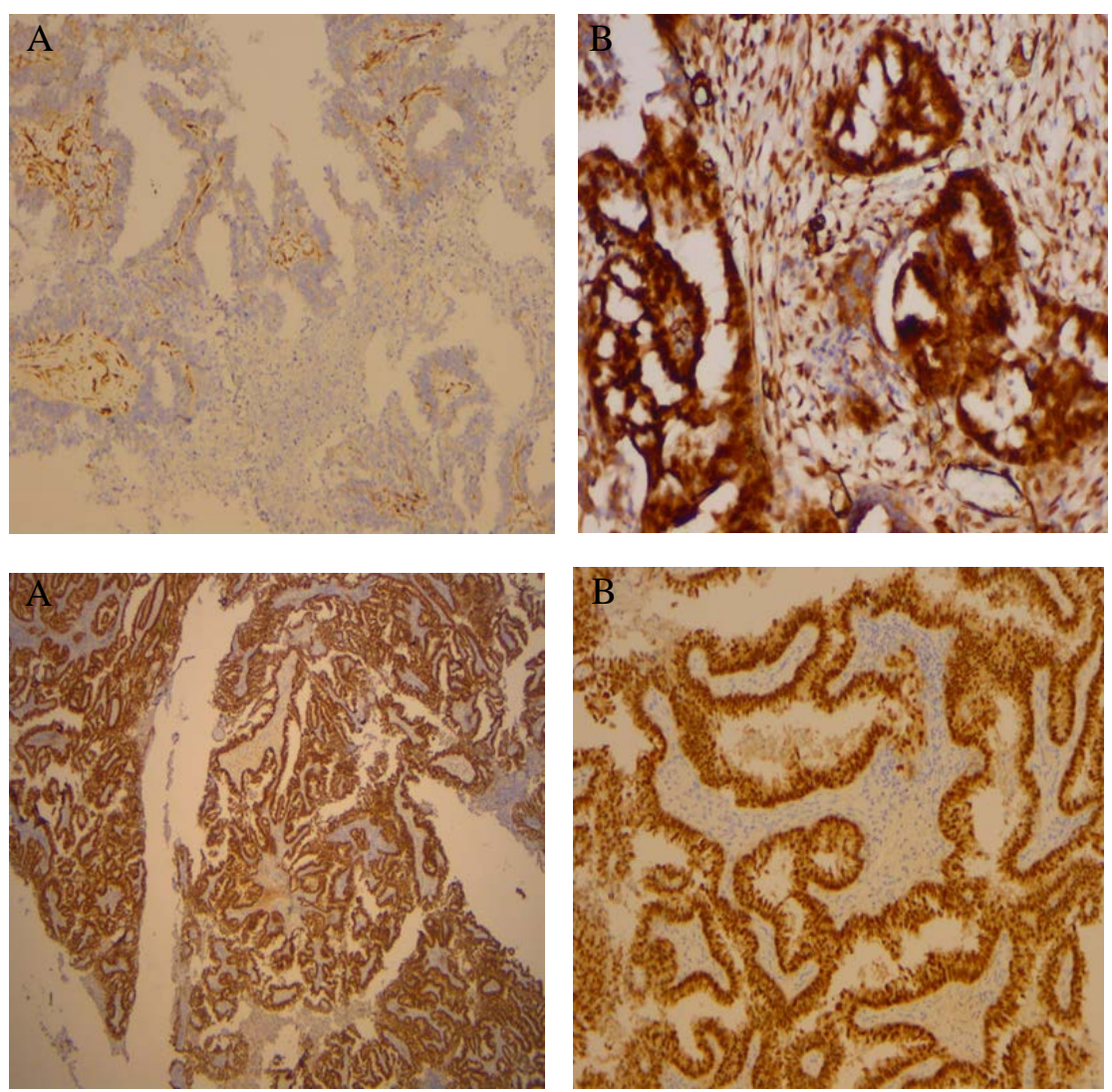

Figure 7. a. X100, b. X200: Immunostaining (IHC) for WT1. a. Negative immuneexpression in breast tumor, b. Positive immune expression in peritoneal serous carcinoma

Figure 8. a. X25. b. X200: IHC for PAX8. Strong diffuse nuclear and cytoplasmic positive expression in breast tumor in different magnifications

\section{DISCUSSION}

Non-mammary metastases to the breast are very uncommon, ${ }^{7}$ representing $<1 \%$ of all breast tumors and associated with advanced disease and poor prognosis, irrespective of the site of origin. The main morphological clues are unusual histologic patterns, lack of an in-situ component and (in some) extensive lymphovascular involvement. Clinical history is vital, although this may often be the first sign of an extramammary primary tumor. Breast metastases from ovarian/ high grade peritoneal serous carcinoma (HGPSC) are rare, showing morphologic and radiologic similarities with primary papillary breast carcinomas, making the diagnosis challenging, leading to very important consequences concerning treatment and prognosis. Most patients who experience metastatic disease have a known history of advanced stage ovarian or peritoneal carcinoma.

Most breast metastases are from the contralateral breast. There are similarities between clinical findings in patients with primary breast cancer and those with metastases to the breast. ${ }^{1}$ Metastases to the breast occur more often in advanced-stage cancer, in patients in their fifth and sixth decades of life, ${ }^{7}$ but they may also be the first sign of an extramammary primary tumor. ${ }^{8,9}$ Metastatic lesions to the breast tend to be more superficial and less fixed to the surrounding tissues than primary breast lesions, but they can also mimic inflammatory breast cancer. ${ }^{10}$ Microcalcifica- tions may accompany serous carcinoma metastases. ${ }^{11,12}$ A papillary structure with fibrovascular cores or psammomatous calcifications is rarely seen in primary breast carcinomas other than invasive micropapillary carcinomas (IMPC). Studies have shown that $85 \%$ of patients present with a solitary tumor, with more common location the upper outer quadrant (62\%). ${ }^{13}$ On mammography, metastatic lesions may appear as discrete, well-circumscribed masses.

Metastatic ovarian carcinoma to the breast (MOCB) is a rare manifestation of recurrent ovarian/peritoneal cancer, with 110 cases reported in the literature until 2016. ${ }^{14}$ Since then, an additional four cases of metastatic primary peritoneal high-grade serous carcinoma to the breast have been reported in previous studies. ${ }^{15-17}$ The typical morphologic features of MOCB include a localized mass with microlobulated margins and posterior enhancement on ultrasound and a well-circumscribed growth pattern surrounded by a fibrous pseudo-capsule, with clear absence of an in situ component. ${ }^{14}$

Primary peritoneal serous carcinoma (PPSC) is morphologically and phenotypically similar to ovarian carcinoma (OC), ${ }^{4}$ and usually spreads superficially in the peritoneal cavity. It also metastasizes to the liver, lung, brain. Breast metastases from PPSC have been scarcely reported. ${ }^{4}$ It is unknown how 
PPSC metastasizes to the breast. It may spread from both lymphatic and hematogenous routes to seed in some organs first, such as colon and liver, and finally metastasizes to the breast. Breast metastasis from a primary ovarian tumor is diagnosed, on average, two years after the initial diagnosis of ovarian cancer. ${ }^{18} \mathrm{~A}$ rare case of PPSC with breast metastasis in a patient 10 years after total abdominal hysterectomy and bilateral salpingo-oophorectomy has been described in the literature. ${ }^{13}$ Likewise, a case of primary peritoneal serous papillary carcinoma that metastasized to an axillary LN, with previous contralateral mastectomy and chemotherapy for carcinoma of the breast, has been described in the literature. ${ }^{19}$ The finding of isolated distant metastatic axillary lymphadenopathy without remarkable intrabdominal disease of primary peritoneal carcinoma has been described in the literature, misinterpreted as primary breast carcinoma. ${ }^{20}$

The length of survival for patients with PPSC and breast metastasis is around 2 to 31 months, ${ }^{5}$ with a five-year survival rate ranging from $0 \%$ to $26,5 \%{ }^{21}$ Our patient, although showing a good response to therapy, succumbed from unrelated causes (rupture of thoracic aortic aneurysm).

In cases of SCs, the papillary architecture and psammoma bodies are characteristic features, indicative of the correct diagnosis. Nevertheless, the appearances of the breast metastases can mimic primary breast tumors. In these cases, the knowledge of the clinical history, the histological comparison of the mammary and extra-mammary malignant tumors, and a broad panel of organ- or tumor-specific immunohistochemical markers, are essential for correct diagnosis. In ER-positive papillary carcinomas, high grade serous ovarian/fallopian tube/peritoneal carcinoma should be considered in the differential diagnosis. In ER/CK7-positive papillary carcinoma, PAX8 and WT1+ expression, are in favor of serous (papillary) carcinoma. Breast-related immunohistochemical markers (GATA-3, GCDFP-15, mammaglobin), could be of help, although not entirely sensitive and specific. PSC and BC share many immunohistochemical profiles (e.g., ER, PR, HER2/neu, CA125, CK7). GCDFP-15/BRST2 and mammaglobin have low sensitivity for $\mathrm{BC}$, thereby limiting their diagnostic utility.

GATA binding protein 3 (GATA 3) immunohistochemistry is primarily used as a marker of breast and urothelial differentiation, particularly in metastatic settings. In the gynecologic tract, it also serves as a robust marker for mesonephric and trophoblastic tumors. GATA-3 shows focal weak to moderate expression in a subset of endometrial and ovarian carcinomas. High-grade serous carcinomas of the gynecologic tract most commonly display a pattern of positivity for PAX8, p53, p16, CK7, WT-1 and variable estrogen receptor and Ki-67 expression. WT1 (Wilm's tumor-1) and PAX8 (Paired-box-gene8) appear to have the greatest utility in differentiating primary BC from metastatic SC, due to their high sensitivity and low potential for aberrant expression. ${ }^{22}$ WT1 is detected in $94.7 \%$ of ovarian (serous) carcinomas, $100 \%$ peritoneal serous carcinomas and 2-3\% IMPC (primary breast invasive micropapillary carcinoma), thereby limiting its utility. ${ }^{23}$ PAX8, a transcription factor expressed in tumors of renal, Mullerian and thyroid origin, is present in 99\% of serous ovarian carcinomas and absent in all BCs, including IMPC. Thus, a positive PAX8 result readily excludes primary BC. ${ }^{24,25}$ Overall, some overlap in IHC analysis may exist between primary breast and gynecologic malignancies, where PAX8 positivity will definitely confirm non-mammary origin of the tumor, as it has not been reported to stain positively in primary breast cancer.

Metastatic high-grade serous carcinomas of the uterine adnexa with BRCA1 deficiency (HGSC-BRCA) have been described to demonstrate characteristic histopathologic features, with pushing/circumscribed patterns of invasion with combinations of solid, pseudo-endometrioid, transitional-like (SET) architecture, high mitotic indices, geographic necrosis and tumor infiltrating lymphocytes. ${ }^{2}$ Metastases with infiltrative invasion patterns show papillary and/or micropapillary architecture. ${ }^{26}$

EPC is a distinct breast histologic entity with particular histomorphologic features, in detail defined in the $5^{\text {th }}$ WHO edition of Breast Tumors. ${ }^{1}$ EPC usually consists of a papillary mass within a cystic space and less often of an aggregate of close nodules. The tumor has a rounded, pushing border and is typically surrounded by a fibrous capsule of varying thickness. There are also cases featuring multiple PClike nodules lacking peripheral fibrous capsules and ME cells and showing irregular outlines. ${ }^{27}$ High grade lesions are extremely rare and should be classified as invasive disease. ${ }^{1}$ EPCs express ER and (usually) PR, lack ERBB2 amplification, and demonstrate a low to occasionally moderate Ki-67 proliferation. Lesions presenting with a growth pattern similar to that of EPC, but with nuclear pleomorphism and increased mitotic activity, and/or a triple-negative or HER-2 positive phenotype, should be graded, staged and managed as IBCs. ${ }^{1}$

Metastasis from serous OC and/or peritoneal carcinoma, in the form of EPC-like, has not been described in the literature. The tumor in our case had the appearance of 'encapsulated cystic papillary carcinoma', arranged in multiple confluent multinodular patterns, with high nuclear grade and brisk mitotic activity. The appearances could be interpreted as 'pushing pattern of invasion'. Interestingly, calci- 
fications have rarely been reported in breast metastases, with the exception of metastatic HGSC. The presence of calcifications (psammoma-like) is consistent with primary papillary lesions of the breast, especially IMPC and may also accompany SC metastases. The architecture of the primary peritoneal serous papillary carcinoma, in our case, did not include EPClike areas.

At the time of breast tumor diagnosis, the clinical history was not known. Although the possibility of metastatic origin should be considered in all papillary lesions of the breast (particularly of the invasive micropapillary pattern), in our case the clinical/surgical approach as of a 'primary' breast tumor (total mastectomy and SLNB), the EPC-pattern of growth (never described before among metastatic patterns) and the diffuse positivity of hormone receptors (luminal B phenotype/increased Ki-67) were more consistent with high-grade EPC. Although most EPCs are recognized to be of low and intermediate grade, a distinct proportion of these tumors do show high cytonuclear grade features in a frequency ranging from $2.5 \%$ to $14 \%$. Nevertheless, in cases of highgrade EPC carcinomas, we should always consider the possibility of metastatic papillary carcinoma, especially of serous and gynecologic origin, even if the diagnosis has not been confirmed. The request for clinical history and IHC staining for PAX8 are mandatory in these cases.

\section{REFERENCES}

1. WHO Classification of Breast Tumours, $5^{\text {th }}$ edition. 2019. ISBN: 978-92-832-4500-1.

2. Rakha EA, Varga Z, Elsheik S, et al. High-grade encapsulated papillary carcinoma of the breast: an under-recognized entity. Histopathology. 2015;66 (5):740-6. doi: 10.1111/his.12591.

3. Liu X, Wu H, Teng L, et al. High-grade encapsulated papillary carcinoma of the breast is clinicpathologically distinct from low/intermediate grade neoplasms in Chinese patients. Histol Histopathol. 2019;34(2):137-47. doi: 10.14670/HH-18-026. Epub 2018 Jul 13.

4. Sun J-Y, Gebre W, Dong Y-M, Shaun X, Robbins R, Podruma A. Primary peritoneal carcinoma metastasizing to breast: a single case report and literature review from clinic to biology. Cancer Biol Med. 2016;13(3):389-95. doi: 10.20892/j.issn.20953941.2016.0058.

5. Recine MA, Deavers MT, Middleton LP, Silva EG, Malpica A. Serous carcinoma of the ovary and peritoneum with metastases to the breast and axillary lymph nodes: A potential pitfall. Am Surg Pathol. 2004;28:1646-51. doi: 10.1097/00000478-200412 000-00015.

6. Khalifeh I, Deavers MT, Christofanilli M, Coleman RL, Malpica A, Gilcrease MZ. Primary peritoneal

\section{CONCLUSION}

Ovarian or peritoneal metastases to the breast are rare and can be the first sign of disease recurrence, misinterpreted with primary breast carcinoma. Metastatic papillary serous carcinoma should be considered in cases of high-grade encapsulated papillary carcinoma of the breast. The correct diagnosis requires a combination of the clinical history, careful clinical examination, radiology and anatomic pathological evaluation. Even in cases of unknown clinical history, positive expression for PAX8 and WT1 is essential to prevent misdiagnosis, ensure accurate treatment and prevent unnecessary surgical intervention. PAX8 seems to be the most useful marker for differentiating OC or PPSC from breast carcinoma, as it has not been reported to stain positively in primary breast cancer. Physicians' awareness of this clinical entity is paramount in allowing early recognition and the initiation of appropriate therapy and optimizing patient outcomes.

\section{ETHICAL CONSIDERATION}

The patient signed a written informed consent to present the medical history and records in this research journal.

\section{CONFLICT OF INTEREST}

None. serous carcinoma presenting as inflammatory breast cancer. Breast J. 2009;15:176-81. doi:

10.1111/j.1524-4741.2009.00693.x.

7. Bombonati A, Lerwill MF. Metastases to and from the breast. Surg Pathol Clin. 2012,;5(3):719-47. doi: 10.1016/j.path.2012.06.004.

8. Buisman FE, van Gelder L, Menke-Pluijmers MB, Bob H. C. Bisschops B.H.C, Peter W. Plaisier P.W, and Westenend P.J: a single institution's experience of a diagnostic challenge with important therapeutic consequences-a retrospective study. World J Surg Oncol. 2016;23;14(1):166. doi: 10.1186/s12957-0160915-4.

9. Lee AH. The histological diagnosis of metastases to the breast from extramammary malignancies. J Clin Pathol. 2007;60(12):1333-41. doi: 10.1136/jcp.2006. 046078.

10. Alvarez RH, Gong Y, Uedo NT, et al. Metastasis in the breast mimicking inflammatory breast cancer. J Clin Oncol. 2012;1;30(22):e202-6. doi: 10.1200/ JCO.2011.40.2230.

11. Lee S H, Park J M, Kook S H, B K Han, W K Moon. Metastatic tumors to the breast: mammographic and ultrasonographic findings. J Ultrasound Med. 2000;19(4):257-62. doi: 10.7863/jum.2000.19.4.257. 
12. Williams SA, Ehlers RA, $2^{\text {nd }}$, Hunt $\mathrm{KK}$, et al. Metastases to the breast from nonbreast solid neoplasms: presentation and determinants of survival. Cancer. 2007;15;110(4):731-7. doi: 10.1002/cncr.22835.

13. Watson GA, Greally M, Murphy DJ, Doyle A, Quinn C, Walshe JM. A rare case of metastatic peritoneal adenocarcinoma presenting as breast cancer. Oncol Cancer Case Rep. 2016;2(2):1-3. doi:10.15436/23770902.16.1014.

14. Tempfer C.B, El Fizazi N, Ergonenc H, Solass W. Metastasis of ovarian cancer to the breast: A report of two cases and review of the literature. Oncology Letters. 2016;11:4008-12. doi:10.3892/ol.2016.4514.

15. Harada Y, Kubo M, Kai M, Yarrada M, Zaguirre K, Obgarri T, Yahata H, Ohishi Y, Yamamoto H, Oda $\mathrm{Y}$ and Nakamura M. Breast metastasis from pelvic high-grade serous adenocarcinoma: a report of two cases. Surgical Case Reports. 2020;6:317-25. doi: 10.1186/s40792-020-01090-7.

16. Park S, Cho E.Y, Oh Y.L, Park Y.H and Kim H-S. Primary Peritoneal High-grade Serous Carcinoma Misinterpreted as Metastatic Breast Carcinoma: A Rare Encounter in Peritoneal Fluid Cytology. Anticancer Research. 2020;40(5):2933-9. doi: 10.21873/anticanres.14271.

17. Girgin G.S, Burcin R. Breast Metastasis of Primary Peritoneal Carcinoma Demonstrated on FDG PET/CT. Clinical Nuclear Medicine: 2021;46(9):pe473-74. doi: 10.1097/RLU.000000000 0003642.

18. Ozguroglu M, Ersavasti G, lvan S, et al. Bilateral inflammatory breast metastases of epithelial ovarian cancer. Am J Clin Oncol. 1999;22(4):408-10. doi: 10.1097/00000421-199908000-00018.

19. Nomoto K, Nakajima T, Miwa S, Hayashi S, Tsuneyama K. Primary peritoneal serous papillary carcinoma that metastasized to an axillary lymph node in a woman with a history of breast cancer: A case report and diagnostic pitfalls. Human Pathology:
Case Reports. 2015;2:90-93. doi: 10.1016/j.ehpc. 2015.03.003.

20. Berker B, Ortac F, Ataoglu O. Axillary lymphadenopathy as the primary presentation of primary peritoneal carcinoma. Gynecol Obstet Invest. 2002;54:232-236.doi: 10.1159/000068387.

21. Eltabbakh GH, Werness BA, Piver S, Blumenson LE. Prognostic factors in extraovarian primary peritoneal carcinoma. Gynecol Oncol. 1998;71:230-9. doi: 10.1006/gyno.1998.5090.

22. Goldstein NS, Bassi D, Uzieblo A. WT1 is an integral component of an antibody panel to distinguish pancreatobiliary and some ovarian epithelial neoplasms. Am J Clin Pathol. 2001;116:246-252. doi: 10.1309/8X4T-35B7-7529-QE7X.

23. Mhawech-Fauceglia P, Kay B, Li C.J, Lin Y.G. Metastatic ovarian papillary serous carcinoma to the breast: Diagnosis and pitfalls. Gynecologic Oncology Reports. 2013;4:35-37. doi: 10.1016/j.gynor. 2012.12.008.

24. Lotan T.L, Ye H, Melamed J, Wu X-R, Shih I-M, Epstein J.I. Immunohistochemical panel to identify the primary site of invasive micropapillary carcinoma. Am J Surg Pathol. 2009;33(7):10371041. doi: 10.1097/PAS.0b013e3181962dcd.

25. Nonaka D, Chiriboga L, Soslow RA. Expression of PAX8 as a useful marker in distinguishing ovarian carcinomas from mammary carcinomas. Am J Surg Pathol. 2008;32:1566-71. doi: 10.1097/PAS.0b0 13e31816d71ad.

26. Reyes M.C, Arnold A.G, Kauff N.D, Levine D.A. Invasion patterns of metastatic high-grade serous carcinoma of ovary or fallopian tube associated with BRCA deficiency. Modern Pathology. 2014;27: 1405-11. doi: 10.1038/modpathol.2013.237.

27. Rakha E.A \& Ellis I.O. Diagnostic challenges in papillary lesions of the breast. Pathology. 2018;50(1):100-10. doi: 10.1016/j.pathol.2017.10. 005. 